\title{
Bell model with hidden variables and Bohr quantum postulate on the existence of indivisible quantum of action given by Planck constant $h$.
}

\author{
Andrei Khrennikov \\ Linnaeus University, International Center for Mathematical Modeling \\ in Physics and Cognitive Sciences Växjö, SE-351 95, Sweden
}

July 18, 2020

\begin{abstract}
This note is a part of my efforts for getting rid of nonlocality from quantum mechanics (QM). Quantum nonlocality is two faced Janus, one face is apparent quantum mechanical nonlocality (assigned with projection postulate), another face is nonlocality of Bell's model with the hidden variables. This paper is directed against the latter. The main casualty of Bell's model is that it contradicts to the Heinsenberg's uncertainty and Bohr's complementarity principles. The aim of this note is to point to the physical seed of the aforementioned principles. This is the quantum postulate: the existence of indivisible quantum of action given by the Planck constant. Bell's model by contradicting to the basic principles of QM implies rejection of this postulate. Thus, it contradicts not only to the QM-formalism, but also to the fundamental feature of the quantum world that was initially discovered by Planck.

keywords: Complementarity and uncertainty principles, indivisible quantum of action, quantum postulate, Copenhagen interpretation, quantum nonlocality, hidden variables.
\end{abstract}

\section{Introduction}

This is the "light version" of the preprint [1] that was overloaded by diverse themes on quantum foundations. After reading the present "light version", the reader can turn to the original paper [1]. 
The aim of this paper is to show that the Bell's model with hidden variables [2]-[5] contradicts to the quantum postulate [6]-[9] - the existence of indivisible quantum of action $h .{ }^{1}$ Thus, the following natural question arises:

Would one like to "explain" the long distance correlations by the cost of confronting again with the ultraviolet (Rayleigh-Jeans) catastrophe and rejecting the original Planck work on the black body radiation?.

This paper is a part of the series of my works which can be unified by the slogan "getting rid of nonlocality from quantum physics" [10][13]. The main message of aforementioned papers is that quantum theory is local. One of complications in getting rid of nonlocality from QM is that so-called "quantum nonlocality" is two faced Janus [14]. People freely refer to his different faces, mix them, and often cannot distinguish them. Two faces of nonlocality Janus are

- Apparent nonlocality of QM based on the projection postulate and discussed in the EPR-paper [15];

- Nonlocality of the Bell model with hidden variables based on misleading interpretation of violation of the Bell inequalities.

In paper [10], violation of the Bell inequalities was treated in the purely quantum framework, i.e., without coupling to hidden variables (see also [16]-[26] and references in [16]).

What does quantum theory say about (non)violation of the Bell inequalities?

As was demonstrated in [10], violation vs. satisfaction of such inequalities is equivalent to local incompatibility vs. compatibility of observables. $^{2}$ These inequalities should be treated as statistical tests for the complementarity principle.

However, a typical representative of the quantum community says that the genuine quantum approach to the Bell inequalities [16]-[26], [10]-[13] is not interesting. The essence of these inequalities is in their derivation on the basis of the Bell model with hidden variables [2][5] and searching for explanation of precise quantum correlations (cf. with critical studies [27]-[33]). In this paper, we question this position

\footnotetext{
${ }^{1}$ Bohr stressed [6] that the essence of quantum theory "may be expressed in the so-called quantum postulate, which attributes to any atomic process an essential discontinuity, or rather individuality, completely foreign to the classical theories and symbolised by Planck's quantum of action."

${ }^{2}$ In the mathematical formalism, this is expressed in the form of noncommutativity of Hermitian operators representing quantum observables, this point was presented already in the pioneer works of Griffiths [16, 20] defending quantum locality.
} 
by showing that the Bell model contradicts not only to the quantum formalism, but also to the basic feature of the quantum physical world, the quantum postulate.

During his life Bohr presented a variety of versions of the complementarity principle. In papers $[12,14,34]$, I expressed my vision on Bohr's ideas as the block of sub-principles (see section 7). I think that such a compact formulation of Bohr's principles is important for further discussions of the type "Bohr vs. Bell" [12]. Nowadays, the Bohr complementarity principle is discussed mainly by philosophers, e.g., in [35, 36, 37]; see, however, e.g., Jaeger at al. [38] for technical studies.)

We start with the comparative analysis of the views of Einstein, Podolsky, and Rosen and Bell (sections 2, 3). Typically one considers Bell as the follower of EPR and claims that the Bell inequality is straightforwardly related to the EPR-pradox. It seems that this viewpoint is misleading. Then we point (section 4) that by considering Bell's hidden variables model, one struggles against the complementarity principle and consequently against the existence of the Planck quantum of action.

Now, I shortly remind the stages of my struggle with quantum nonlocality. In [10], it is was shown that violation of Bell-type inequalities can be mathematically formulated in terms of commutators of local observables. By quantum theory noncommutative observables are known as incompatible. Thus, violation of Bell's inequality is a consequence of the Bohr's complementarity principle. Therefore, even formulation of the Bell's model with hidden variables contradicts to the basic principles of QM. This was the output of paper [10]. However, in principle, one still can say: What is the matter? May be we can violate these basic principles of $\mathrm{QM}$ and even abandon the mathematical formalism of QM. (Of course, in the light of tremendous success of quantum theory such a position is very shaky.) In this note, we show that by abandoning the complementarity and uncertainty principles, one confronts with the most fundamental feature of quantum world, the existence of indivisible quantum of action $h$.

\section{EPR}

We start from the commonly forgotten historical fact that the EPR paper was directed against the Copenhagen interpretation of the wave function [15]. Since this interpretation has many versions (Plotnitsky even proposed to speak about interpretations in the spirit of Copenhagen $[35,36])$, it is important to specify the EPR treatment of this interpretation. 
Copenhagen interpretation (EPR) Wave function (quantum state) $\psi$ represents the state of an individual quantum system.

It is important to stress that "state" is interpreted epistemically as representing knowledge about possible outcomes of measurements on the system in the state $\psi$. So, $\psi$ is not the ontic state - not the state of the system as it is, i.e., without relation to external observations. State's interpretation in the EPR-paper is very close the modern information interpretations used in quantum information theory. This point has not been so much highlighted (see, however, $[39,40]$ ).

By this interpretation the quantum mechanical description based on the wave function representation of the state of a quantum system is complete. The complete physical theory is defined as follows [15]: any element of physical reality have a counterpart in the physical theory.

The EPR-reasoning was based on two basic quantum mechanical principles:

- reduction of the wave function (the projection postulate) resulting from measurement's back-action;

- the Heisenberg uncertainty principle.

The latter was formulated as follows: "It is shown in quantum mechanics that, if the operators corresponding to two physical quantities, say $A$ and $B$, do not commute, $A B \neq B A$, then the precise knowledge of one of them precludes such a knowledge of the other. Furthermore, any attempt to determine the latter experimentally will alter the state of the system in such a way as to destroy the knowledge of the first."

EPR showed that the assumption that QM (endowed with the Copenhagen interpretation) is a complete theory implies violation of the Heisenberg uncertainty principle. Since they were sure in validity of this principle, EPR concluded that the quantum mechanical description of nature is incomplete.

We emphasize that EPR did not question the validity of the Heisenberg principle (see appendix). If it would be possible to violate this principle, then assigning to the same system two wave functions which are eigenfunctions of observables represented by non-commutative operators would not lead to any problem (see appendix).

Thus, by concluding that "... the wave function does not provide a complete description of the physical reality, we left open the question of whether or not such a description exists" and believing "...that such a theory is possible", they do not dream for a theory violating the Heisenberg's uncertainty principle. By reading later works of Einstein we can guess that he wanted to construct a classical field model underlying QM [41].

We remark that EPR did not question validity of quantum mechanical description, they were just looking for a more detailed description. But, this deeper description should respect the basic principles of QM, including the uncertainty and complementarity principles. 


\section{Bell}

Although Bell started his paper [2] with referring to the EPR paper as proving incompleteness of QM, his model with hidden variables has not so much to do with the EPR-dream for a complete physical theory generalizing QM. It is surprising that this inconsistency has never been emphasized in numerous papers on Bell's inequality (see, e.g., Aspect [42, 43]). The main difference of Bell's model from the EPR-dream is that his model is in the striking contradiction with the quantum mechanical description, especially with the Heisenberg uncertainty principle (see appendix).

Consider Bell's random variables $A(a, \lambda), B(b, \lambda)$ representing observables of Alice and Bob, respectively. Surprisingly, Bell did not highlighted that, besides probabilities

$$
p_{a, b}(x, y)=p(A(a, \lambda)=x, B(b, \lambda)=y)
$$

for compatible observables, Bell's model describes probabilities

$$
p_{a, a^{\prime}}\left(x_{1}, x_{2}\right)=p\left(A(a, \lambda)=x_{1}, A(a, \lambda)=x_{2}\right)
$$

for generally incompatible observables represented by noncommuting operators. (This problem is especially clear in consideration of CHSH-inequality [5].) From the very beginning, i.e., without any Bell's type inequality, this assumption contradicts to QM-representation of observables and, hence, to the Heisenberg uncertainty principle (or more generally to the Bohr complementarity principle).

Of course, Bell may proceed with his special class of subquantum models, but without identification of the values of his random variables with values of quantum observables and without identification of "hidden correlations" with the experimental correlations. (De Broglie emphasized [44] this viewpoint.) But, Bell wanted experimental verification...

Thus, from the very beginning Bell's model of hidden variables was designed as contradicting the uncertainty principle. Therefore, it is not surprising that, as was shown in my recent paper [10], violation-satisfaction of the CHSH-inequality can be formulated in terms of noncommutativitycommutativity of operators representing local observables of Alice and Bob, respectively.

\section{Crusade against complementarity}

The Heisenberg uncertainty principle was the starting point for Bohr's formulation of the complementarity principle [6]-[8] (see my recent papers [12, $10,34]$ for non-philosophers gently presentation of this principle, see also section 7). Thus, in the light of above consideration, we can say that in fact Bell's argument was directed against the Bohr complementarity principle. This Crusade against complementarity was overshadowed by nonlocality issue. Of course, rejection of the complementarity principle (or the Heisenberg uncertainty principle) would have similar catastrophic consequences even for non-compound systems, say a single atom or neutron, as we can see from the contextuality tests (see, e.g., [45]). 
To discard the Bell model with hidden variables, one need not to derive inequalities and test them experimentally. Of course, one should believe in the basic principles of QM. (In the opposite case, she should say explicitly about this, about her battle against the quantum postulate.) The main impact of experimental tests [46]-[50] is demonstration that quantum correlations (predicted by QM) are preserved for long distances. The latter plays the crucial role in quantum engineering. However, correlations preservation can be checked directly without inequalities. Moreover, by operating with say CHSH-combination of correlations experimenter can miss mutual compensation of deviations from QM. In Aspect's pioneer experiment [51], correlations did not match the quantum prediction, but they mystically compensated each other to violate the Bell inequality (see [52] for discussion). (In spite of numerous discussions with experimenters, I am still not sure that data from the basic experiments on say $\mathrm{CHSH}$-inequality is clean from the mentioned Aspect-type anomaly. Papers typically present only the CHSHcorrelation combination, but not separate correlations for pairs of experimental settings.)

\section{Explaining: long distance correla- tions vs. violation of complementarity principle}

Typically, followers of the Bell argument (that has not so much to do with the original EPR-argument) say they want to explain the long distance correlations. I think that the essence of the problem is in the word "explain".

In science, we operate with mathematical models of physical processes. So, "explain" means "to describe by some mathematical model". And quantum mathematics, as a mathematical model, describes perfectly the long distance correlations: entangled states and projection type measurements. So, it seems that Bell and his followers have something different in mind.

Why was Bell not satisfied with the quantum mechanical description?

From reading Bell, I have the impression that he "simply" wanted to re-establish realism of classical physics. But, what is the main quantum barrier for such realism? Everybody knows this very well, this is the Bohr complementarity principle with starting point at the Heisenberg uncertainty relation; see Bohr [6], "... an independent reality in the ordinary physical sense can neither be ascribed to the phenomena nor to the agencies of observation." This is clearly stated in the EPR-paper [15]. Bell and his followers have to say something as following: we want to break the Heisenberg uncertainty relations. Unfortunately, it was never stated explicitly. Instead, people operate with such an ambiguous notion as "local realism".

Suppose somebody, say Alice, questions the Heisenberg uncertainty principle. Then, why should she consider compound systems? Does she think that these principle is violated only for compound systems? It would be really strange. Thus, before trying to explain the long distance correlations with the Bell-type hidden variables model, it would be reasonable to try 
explain incompatibility of observables corresponding spin projections to different axes or incompatibility of position and momentum observables.

The main feature of the Bell model with hidden variables, the feature crying for justification, is violation of the complementarity principle. It is not so natural to try to "explain" long distance correlations without any attempt to explain violation of this principle.

\section{The root of complementarity: Devil is in the Planck constant}

By starting the anti-complementarity battle, it is useful to remind the foundational roots of complementarity. The Bohr's complementarity principle will be discussed in detail in section 7 .

For Bohr, the root of the complementarity is the existence of indivisible quantum of action given by the Planck constant $h$. The existence of this quantum prevents separation of the genuine physical features of a system from the features of interaction with a measurement apparatus. So, the seed of the Bohr complementarity principle is the Planck constant $h$.

It is meaningless to start a Crusade against complementarity without trying to understand the origin of this fundamental quantum of action in nature. Neither Einstein nor Bell tried to perform such investigation; in fact, neither Bohr nor Heisenberg, for them this is just the feature of nature such as, e.g., the constancy of light's velocity $c$. And, for the moment, this position can be considered as the only possible.

\section{Bohr's complementarity principle}

In 1949, Bohr [9] presented the essence of complementarity in the following widely citing statement:

"This crucial point ... implies the impossibility of any sharp separation between the behavior of atomic objects and the interaction with the measuring instruments which serve to define the conditions under which the phenomena appear. In fact, the individuality of the typical quantum effects finds its proper expression in the circumstance that any attempt of subdividing the phenomena will demand a change in the experimental arrangement introducing new possibilities of interaction between objects and measuring instruments which in principle cannot be controlled. Consequently, evidence obtained under different experimental conditions cannot be comprehended within a single picture, but must be regarded as complementary in the sense that only the totality of the phenomena exhausts the possible information about the objects."

By analyzing this Bohr's statement, I propose [12, 10, 34] to present the Bohr complementarity principle as the following five interconnected principles:

- Contextuality: Irreducible dependence of measurement's output on the experimental context. 
- Context complementarity: Existence of complementary experimental contexts.

- Individuality: Discreteness of quantum measurements -generation of physical phenomena.

- Completeness: Complementary observations provide complete information about system's state.

In this formulation, the complementarity principle can be treated as an epistemological principle (see, especially, paper [34] on coupling to quantum information theory).

\section{Concluding remarks}

The ontological seed of the complementarity principle is the quantum postulate. Therefore rejection of complementarity, as was done by Bell in his model with hidden variables, is impossible without rejection of the existence of indivisible quantum of action. By rejection of the latter, we would cast away all physical achievements of 20th century and confront again the problem of black body radiation.

\section{Appendix: EPR vs. Bell in relation to Heisenberg's uncertainty principle}

As was emphasized already in the abstract of EPR-paper [15], EPR did not question Heisenberg's uncertainty principle:

"In quantum mechanics in the case of two physical quantities described by non-commuting operators, the knowledge of one precludes the knowledge of the other. Then either (1) the description of reality given by the wave function in quantum mechanics is not complete or (2) these two quantities cannot have simultaneous reality. Consideration of the problem of making predictions concerning a system on the basis of measurements made on another system that had previously interacted with it leads to the result that if (1) is false then (2) is also false. One is thus led to conclude that the description of reality as given by a wave function is not complete."

It is clear that EPR cannot even imagine that (2), i.e., Heisenberg's uncertainty principle, is false. For them, it was clear that these two quantities (incompatible observables) cannot have simultaneous reality. In Bell's model with hidden variables, quantities $A(a, \lambda)$ and $A\left(a^{\prime}, \lambda\right)$ have simultaneous reality. (Here $a$ and $a^{\prime}$ are orientations of Alice's beam splitter.) One may say that this Bellian reality, a part of local realism, differs from EPR-reality, "the possibility of predicting it with certainty, without disturbing the system." One may say that EPR wrote about experimental predictions, but generally $A(a, \lambda)$ and $A\left(a^{\prime}, \lambda\right)$ are components of some mathematical model, just a possible human image of a causal subquantum model. This is the good point. But, Bell did not proceed in this way. He simply identified the values of random variables of hidden variables with outcomes of quantum observables, 
the real physical observables. The latter couples of Bellian (hidden variables) reality with the EPR (outcome prediction) reality. So, Bell's introduction of hidden variables contradicts even the statement in the abstract of the EPR-paper ...

Finally, I remind that this viewpoint was presented a long ago by De Broglie as his reaction to Bell's inequality [44].

\section{References}

[1] A. Khrennikov, Quantum postulate vs. quantum nonlocality: Is Devil in h? arXiv:2003.05718 [quant-ph].

[2] Bell, J.S. On the Einstein-Podolsky-Rosen paradox. Physics 1964, 1, 195-200

[3] Bell, J.S. Speakable and Unspeakable in Quantum Mechanics, 2nd ed.; Cambridge University Press: Cambridge, UK, 2004.

[4] Bell, J.S. On the problem of hidden variables in quantum theory. Rev. Mod. Phys. 1966, 38, 450.

[5] Clauser, J.F.; Horne, M.A.; Shimony, A.; Holt, R.A. Proposed experiment to test local hidden-variable theories. Phys. Rev. Lett. 1969, 23, 880 .

[6] N. Bohr, The quantum postulate and the recent development of atomic theory. Supplement to Nature, April 14 , 580-590 (1928).

[7] N. Bohr, Wirkungsquantum und Naturbeschreibung. Naturwissenschaft, 17, (1929).

[8] N. Bohr, The quantum of action and the description of nature. In: Foundations of Quantum Physics I (1926-1932). Niels Bohr collected works. J. Kalckar (ed.), v. 6, Elsevier B.V., 1985, pp. 201-217.

[9] Bohr, N. The Philosophical Writings of Niels Bohr; Ox Bow Press: Woodbridge, UK, 1987.

[10] A. Khrennikov, Get rid of nonlocality from quantum physics. Entropy, 21(8), 806 (2019).

[11] A. Khrennikov, Bell argument: Locality or realism? Time to make the choice. AIP Conf. Proc., 1424, pp. 160-175 (2012); https://arxiv.org/pdf/1108.0001v2.pdf

[12] A. Khrennikov, Bohr against Bell: complementarity versus nonlocality. Open Phys. 15, 734-73 (2017).

[13] A. Khrennikov, Quantum versus classical entanglement: eliminating the issue of quantum nonlocality. arXiv:1909.00267v1 [quant-ph].

[14] A. Khrennikov, Two faced Janus of quantum nonlocality. arXiv:2001.02977 [quant-ph]

[15] Einstein, A.; Podolsky, B.; Rosen, N. Can quantum-mechanical description of physical reality be considered complete? Phys. Rev. 1935, 47, $777-780$. 
[16] R. B. Griffiths, Consistent Quantum Theory (Cambridge 2002). http://quantum.phys.cmu.edu/CQT/

[17] De Muynck, W. Foundations of Quantum Mechanics, an Empiricist Approach; Springer: Dordrecht, 2006.

[18] A. Yu. Khrennikov, The role of von Neumann and Luders postulates in the Einstein, Podolsky, and Rosen considerations: Comparing measurements with degenerate and nondegenerate spectra. J. Math.Phys.,49, N 5, art. no. 052102 (2008).

[19] R. B. Griffiths, Quantum locality. Found. Phys. 41 (2011) 705-733.

[20] R. B. Griffiths, Consistent quantum measurements, Stud. Hist. Phil. Mod. Phys. 52 (2015) 188-197.

[21] R. B. Griffiths, What quantum measurements measure, Phys. Rev. A 96 (2017) 032110.

[22] Boughn, S. Making sense of Bell's theorem and quantum nonlocality. Found. Phys. 2017, 4\%, 640-657.

[23] Jung K. Violation of Bell's inequality: Must the Einstein locality really be abandoned? J. Phys.: Conf. Ser. 2017 880, 012065, 1-8.

[24] Griffiths, R.B. Quantum nonlocality: Myth and reality. arXiv 2019, arXiv:1901.07050.

[25] R. B. Griffiths, Nonlocality claims are inconsistent with Hilbertspace quantum mechanics, Phys. Rev. A 101 (2020) 022117, arXiv:1901.07050.

[26] Cetto, A.M., Valdes-Hernandez, A.; de la Pena, L. On the spin projection operator and the probabilistic meaning of the bipartite correlation function. Found Phys (2020) 50, 27-39.

[27] Kupczynski, M. Bertrand's paradox and Bell's inequalities. Phys. Lett. A 1987, 121, 205-207.

[28] Khrennikov, A. Non-Kolmogorov probability models and modified Bell's inequality, J. Math. Phys. 2000, 41, 1768-1777.

[29] Khrennikov, A. Yu. Interpretations of Probability; VSP Int. Sc. Publishers: Utrecht/Tokyo, 1999; 2nd edn., De Gruyter: Berlin, 2009.

[30] Nieuwenhuizen, T. M. Is the contextuality loophole fatal for the derivation of Bell inequalities? Found. Phys. 2011, 41, 580-591.

[31] De Raedt, H.; Hess, K.; Michielsen, K. Extended Boole-Bell inequalities applicable to quantum theory. J. Comp. Theor. Nanosc. 2011, 8, 10119.

[32] Khrennikov, A. CHSH inequality: quantum probabilities as classical conditional probabilities. Found. Phys. 2015, 45, 711-725.

[33] Khrennikov, A.; Alodjants, A. Classical (local and contextual) probability model for Bohm-Bell type experiments: no-signaling as independence of random variables, Entropy 2018, 21, 157.

[34] A. Khrennikov, Echoing the recent Google success: Foundational roots of quantum supremacy. arXiv:1911.10337 [quant-ph]. 
[35] Plotnitsky, A. Epistemology and Probability: Bohr, Heisenberg, Schrödinger and the Nature of Quantum-Theoretical Thinking; Springer: Berlin, Germany; New York, NY, USA, 2009.

[36] Plotnitsky, A. Niels Bohr and Complementarity: An Introduction; Springer: Berlin, Germany; New York, NY, USA, 2012.

[37] J. Faye, H. Folse, Niels Bohr and the Philosophy of Physics: TwentyFirst-Century Perspectives. Bloomsbury Academic, 2017.

[38] G. Jaeger, A. Shimony, and L. Vaidman, Two interferometric complementarities. Phys. Rev. A 51, 54 (1995).

[39] C.F. von Weizsäcker, Aufbau der Physik. Hanser Verlag, München 1985 .

[40] A. Plotnitsky, "Without in any way disturbing the system": Illuminating the issue of quantum nonlocality. arXiv:1912.03842 [quant-ph].

[41] Einstein, A. and Infeld, L. (1938). The Evolution of Physics: The Growth of Ideas from Early Concepts to Relativity and Quanta, Cambridge, Cambridge University Press.

[42] A. Aspect, Experimental tests of Bell's inequalities in atomic physics, in Atomic Physics 8, Proceedings of the Eighth International Conference on Atomic Physics, edited by I. Lindgren, A. Rosen and S. Svanberg (1982).

[43] A. Aspect, Bell's Theorem: The naive view of an experimentalist. quant-ph/0402001.

[44] De Broglie, L. (1964). The current interpretation of wave mechanics: a critical study. Elsevier.

[45] H. Bartosik, J. Klepp, C. Schmitzer, S. Sponar, A. Cabello, H. Rauch, Y. Hasegawa, Experimental test of quantum contextuality in neutron interferometry. Phys. Rev. Lett. 103 (2009) 040403.

[46] Aspect, A.; Dalibard, J.; Roger, G. Experimental test of Bell's Inequalities using time-varying analyzers. Phys. Rev. Lett. 1982, 49, 18041807.

[47] G. Weihs et al., Violation of Bell's inequality under strict Einstein locality conditions. Phys. Rev. Lett. 81, 1998, S. 5039.

[48] Hensen, B.; Bernien, H.; Dreau, A.E.; Reiserer, A.; Kalb, N.; Blok, M.S.; Ruitenberg, J.; Vermeulen, R.F.; Schouten, R.N.; Abellan, C.; et al. Experimental loophole-free violation of a Belli nequality using entangled electron spins separated by $1.3 \mathrm{~km}$. Nature 2015, 526, 682 .

[49] Giustina, M.; Versteegh, M.A.; Wengerowsky, S.; H.; steiner, J.; Hochrainer, A.; Phelan, K.; Steinlechner, F.; Kofler, J.; Larsson, J.Å; Abellan, C.; et al. A significant-loophole-free test of Bell's theo-rem with entangled photons. Phys. Rev. Lett. 2015, 115, 250401.

[50] Shalm, L.K.; Meyer-Scott, E.; Christensen, B.G.; Bierhorst, P.; Wayne, M.A.; Stevens, M.J.; Gerrits, T.; Glancy, S.; Hamel, D.R.; Allman, M.S.; et al. A strong loophole-free test of local realism. Phys. Rev. Lett. 2015, 115, 2504. 
[51] A. Aspect, Trois tests expérimentaux des inégalités de Bell par mesure de corrélation de polarisation de photons, thése d' Etat, Orsay (1983).

[52] G. Adenier, A. Khrennikov, Anomalies in experimental data for the EPR-Bohm experiment: Are both classical and quantum mechanics wrong? arXiv:quant-ph/0607172. 